\title{
DOCTRINA
}

\section{Contaminantes emergentes en el agua: Regulación en México, principio precautorio y perspectiva comparada}

\author{
Emerging contaminants in water: Regulation in Mexico, \\ precautionary principle and comparative perspective
}

\author{
Daniel Jacobo-Marín \\ Universidad de Jaén, España
Germán Santacruz de León iD
El Colegio de San Luis, México

\begin{abstract}
RESUMEN Este trabajo analiza el estado de regulación jurídica de los contaminantes emergentes en las fuentes y cuerpos de agua en México. Para este fin, se debate su relevancia en el contexto de las obligaciones constitucionales asumidas en torno al derecho humano al agua y al saneamiento. Se revisan los instrumentos internacionales y se elabora un breve balance general que permite reflexionar sobre el vacío legal de este tópico en México. Se muestra que los contaminantes emergentes son sustancias de diverso origen y naturaleza química, cuya presencia en el medio ambiente no se considera significativa en razón de su distribución y concentración, por lo que pasan inadvertidos. No obstante, debido al aumento en su detección, el potencial impacto ecológico y los riesgos que entrañan a la salud humana, han recibido atención reciente en análisis académicos, estudios oficiales y políticas de calidad del agua. Se propone, desde la perspectiva del derecho comparado y bajo el enfoque del principio precautorio, un esquema de reglamentación normativa nacional. Con ese propósito, se realiza un análisis comparativo de los marcos jurídicos chileno y europeo. Se concluye que la ausencia de dispositivos que normen los contaminantes emergentes obstaculiza el cumplimiento del derecho humano al agua y al saneamiento, sobre todo el que se refiere a dos aspectos centrales: el adecuado saneamiento y el suministro de agua de calidad para el consumo doméstico.
\end{abstract}

PALABRAS CLAVE Derecho humano al agua, contaminantes emergentes, principio precautorio, calidad de agua, derecho comparado. 


\begin{abstract}
This paper analyzes the status of legal regulation of emerging contaminants in water sources in Mexico. To this end, its relevance is debated in the context of the constitutional obligations assumed regarding the human right to water and sanitation (HRWS). The international instruments are reviewed and a brief general balance is prepared that allows us to reflect on the legal vacuum of this topic in Mexico. Emerging contaminants are shown to be substances of diverse origin and chemical nature, the presence of which in the environment is not considered significant due to their distribution and concentration, so they go unnoticed. However, due to the increase in their detection, the potential ecological impact and the risks they pose to human health, they have received recent attention in academic analysis, official studies and water quality policies. It is proposed, from the perspective of comparative law and under the precautionary principle approach, a scheme of national normative regulation, for that purpose, a comparative analysis is carried out with the Chilean and European legal frameworks. It is concluded that the absence of devices that regulate emerging contaminants hinders compliance with HRWS, especially that which refers to two central aspects: adequate sanitation and the supply of quality water for domestic consumption.
\end{abstract}

KEYWORDS Human right to water, emerging contaminants, precautionary principle, quality of water, comparative law.

\title{
Introducción
}

El propósito de este artículo es analizar el estado actual de estudio e identificación de contaminantes emergentes en el agua (comunes, frecuentes, detectables) en México. Para este fin, se debate su relevancia en el ámbito de los compromisos constitucionales asumidos en torno al derecho humano al agua y al saneamiento. La investigación tiene como contexto la revisión técnico-normativa de las aguas residuales no sometidas a tratamiento y los efluentes de plantas que no están diseñadas para tratar los contaminantes emergentes, por lo que una parte de estos compuestos y sus metabolitos ingresan con una alta porción de toxicidad a las corrientes superficiales, a los acuíferos y a los sistemas marinos, que, por lo general, son fuentes de suministro de agua para las plantas potabilizadoras. Debido a las propiedades físico-químicas de las referidas sustancias - entre ellas, alta solubilidad en el agua y poca biodegradación-, son capaces de llegar a diversos entornos y representar riesgos potenciales para el consumo humano de agua.

Se esboza, como problema jurídico, que el reconocimiento del derecho humano al agua y al saneamiento en el régimen constitucional mexicano el 8 de febrero de 2012 supuso un avance legal, sin embargo, la información oficial sobre agua apta para el consumo humano y el tratamiento de las aguas residuales muestra un panorama poco alentador, sobre todo porque la identificación y la efectiva remoción de contaminantes emergentes son tópicos ausentes en la regulación sobre calidad del agua. En esa tesitura, este trabajo se orienta hacia la siguiente hipótesis: el vacío legal 
en México sobre contaminantes emergentes vulnera el cumplimiento progresivo del derecho humano al agua y al saneamiento, fundamentalmente, el que se refiere a dos aspectos básicos: el saneamiento adecuado y el suministro de agua de calidad para el consumo doméstico, actividades cuya observancia compete a las autoridades de los tres órdenes de gobierno.

Respecto al diseño metodológico, para cumplir el objetivo planteado se elaboraron tres tareas específicas: en primer lugar, una revisión documental y bibliográfica en torno a los conceptos centrales discutidos en este trabajo: el derecho humano al agua, los contaminantes emergentes, el principio precautorio ambiental y la regulación sobre la calidad de las aguas; luego, se realiza un examen legislativo exhaustivo sobre los contaminantes emergentes en México y, finalmente, se aborda un breve análisis comparativo con el fin de ilustrar los rasgos normativos comunes y distintivos sobre el referido tema.

El artículo está organizado en cinco apartados. El primero aborda el derecho humano al agua y al saneamiento en el contexto del debate sobre los contaminantes emergentes; se caracterizan los principales contaminantes y se enfatizan los componentes teórico-normativos del citado derecho, contenidos en los instrumentos internacionales. El segundo analiza los contaminantes emergentes a la luz del principio precautorio, con el fin de debatir la ausencia de regulación sobre estas sustancias en México y las actividades que entrañan peligro a la salud humana y los ecosistemas acuáticos. El tercer apartado contiene, con base en una revisión histórico-normativa, la regulación de las sustancias químicas contaminantes en el régimen jurídico del agua en México, de manera particular, las Normas Oficiales Mexicanas (NOM) sobre calidad y tratamiento. El cuarto examina, desde el derecho comparado, los principales ejes de actuación relativos a la vigilancia y los mecanismos de acción para identificar y tratar las sustancias contaminantes emergentes, concretamente con los marcos normativos de Chile y la Unión Europea. El último apartado corresponde a las conclusiones.

\section{El derecho humano al agua y al saneamiento en el contexto del debate sobre los contaminantes emergentes}

Durante las últimas décadas la comunidad científica, de manera transversal, ha centrado sus esfuerzos en el estudio de los contaminantes químicos, en su mayoría apolares, tóxicos, persistentes y bioacumulables, como los hidrocarburos aromáticos policíclicos, los policlorobifenilos o las dioxinas, cuya presencia, identificación y tratamiento se han regulado en diversas normas y reglamentaciones. Sin embargo, el desarrollo de métodos de análisis más sensibles ha permitido alertar sobre la presencia de contaminantes potencialmente peligrosos, denominados emergentes de forma genérica (Barceló y López de Alda, 2007: 12). 
El concepto contaminantes emergentes se emplea para referirse a compuestos de diverso origen y naturaleza química, cuya presencia en el medio ambiente no se considera significativa en términos de distribución y concentración, por lo que pasan inadvertidos. No obstante, debido al aumento en su detección, del potencial impacto ecológico y los riesgos que entrañan a la salud humana, se han convertido en el foco de atención reciente en análisis académicos, estudios oficiales y políticas de calidad del agua. Los contaminantes emergentes no necesitan permanecer de forma constante en el ambiente para causar efectos negativos debido a que sus tasas de transformación/remoción se pueden compensar por su continua introducción y acumulación (Gil y otros, 2012: 53).

Las aguas residuales no sometidas a tratamiento y los efluentes de plantas que no están diseñadas para tratar este tipo de sustancias son una de las principales fuentes de contaminantes emergentes, por lo que una parte de estos compuestos y sus metabolitos ingresan con una alta porción de toxicidad a las corrientes superficiales, a los acuíferos y a los sistemas marinos, que generalmente son fuentes de suministro de agua para las plantas potabilizadoras. Debido a sus propiedades fisicoquímicas -entre ellas, alta solubilidad en agua y poca biodegradación - son capaces de llegar a diversos entornos y representar riesgos potenciales para el consumo humano de agua. Algunos efectos significativos documentados se refieren a la alteración del sistema endocrino y el bloqueo o perturbación de las funciones hormonales (García, Gortáres y Drogui, 2011: 97).

La identificación de las aguas residuales como fuente principal de contaminantes emergentes se relaciona con el volumen del flujo que está sujeto al muestreo; en tal caso los contaminantes emergentes son menos persistentes durante temporadas de escorrentía amplia. De forma general, la política de gestión hídrica en las ciudades se ha centrado en el suministro continuo del líquido, de tal modo que las investigaciones tradicionales sobre calidad del agua tienen como objetivo los nutrientes, bacterias, metales pesados y contaminantes prioritarios cuyos efectos en la salud humana han sido registrados, como pesticidas, químicos industriales e hidrocarburos. En las últimas décadas las investigaciones han revelado la existencia de sustancias orgánicas contaminantes que constituyen una amenaza para el ecosistema luego de ser liberadas en los flujos de aguas residuales; estos contaminantes pertenecen a diversas familias químicas y son típicamente detectados en niveles de trazas, es decir, ng/l (nanogramos por litro) o $\mu \mathrm{g} / \mathrm{l}$ (microgramos por litro) (Bai y otros, 2018: 133-137).

El incremento en la fabricación y el consumo de productos farmacéuticos para usos hospitalarios, veterinarios, cosméticos y domésticos, también aumentan, de forma concomitante, la descarga de sustancias activas en los sistemas de drenaje municipales, lo que mantiene la toxicidad de los componentes en los sistemas de desalojo de aguas residuales y los ecosistemas acuáticos. 


\section{El derecho humano al agua y al saneamiento en el derecho internacional}

El derecho humano al agua y al saneamiento se ha construido a lo largo de un proceso de discusión política donde dos de sus elementos centrales -el saneamiento adecuado y la calidad del agua suministrada para el uso y el consumo domésticohan quedado al margen en el debate sobre los contaminantes emergentes. El derecho humano al agua y al saneamiento ha sido abordado en diversos contextos, perspectivas y ámbitos disciplinarios, y fue durante la segunda mitad del siglo XX cuando se ensayaron definiciones oficiales en los instrumentos internacionales (Jacobo-Marín, 2013).

El derecho de acceso al agua para el consumo humano fue reconocido en acuerdos internacionales relativos al tratamiento de los prisioneros de guerra, ${ }^{1}$ a saber: Convenio de Ginebra relativo al trato debido a los prisioneros de guerra (Convenio III) de 1949, Convenio de Ginebra relativo a la protección debida a las personas civiles en tiempo de guerra (Convenio IV) de 1949, Protocolo adicional a los Convenios de Ginebra relativo a la protección de las víctimas de los conflictos armados internacionales (Protocolo I) de 1977 y Protocolo adicional a los Convenios de Ginebra relativo a la protección de las víctimas de los conflictos armados sin carácter internacional (Protocolo II) de 1977 (Jacobo-Marín, 2013: 49-52).

La Convención sobre la Eliminación de Todas las Formas de Discriminación contra la Mujer (18 de diciembre de 1979) se refiere de manera explícita al derecho al agua y al saneamiento (artículo 14, apartado 2, inciso h). Casi exactamente diez años después, el 20 de noviembre de 1989, se aprobó la Convención sobre los Derechos del Niño, en la que se menciona la importancia del agua, el saneamiento ambiental y la higiene para garantizar el desarrollo integral de los infantes, incluyendo los peligros y riesgos de contaminación del medio ambiente y el agua (artículo 24-2). La Conferencia Internacional sobre Agua y Desarrollo Sostenible o Conferencia de Dublín, celebrada en la ciudad irlandesa del 26 al 31 de enero de 1992, estableció en el principio 4: «es esencial reconocer ante todo el derecho fundamental de todo ser humano a tener acceso al agua pura y al saneamiento por un precio asequible». Además, se reconoció en este controversial principio 4 que el agua tiene un valor en todos los usos en los que se destina y debería reconocérsele como un bien económico.

Algunos meses después, en junio de 1992, la Conferencia de las Naciones Unidas sobre el Medio Ambiente y el Desarrollo (Cumbre de Río) refrendó, a través del Programa 21, la resolución de la Conferencia de Mar del Plata sobre el Agua por la que

\footnotetext{
1. Los acuerdos sobre la guerra constituyen un conjunto de reglas producido en el ámbito del derecho militar, que incluye las acciones convencionalmente aceptadas para participar en un conflicto armado (ius ad bellum) y de los límites aceptables de conducta durante los enfrentamientos (ius in bello), éste último derivó, teóricamente, en el llamado derecho internacional humanitario.
} 
se reconocía que todas las personas tienen derecho al acceso al agua potable, lo que se denominó «la premisa convenida». En septiembre de 1994 la Conferencia Internacional de las Naciones Unidas sobre la Población y el Desarrollo estableció, en su Programa de Acción, que toda persona «tiene derecho a un nivel de vida adecuado para sí y su familia, incluidos alimentación, vestido, vivienda, agua y saneamiento».

Una resolución de la Asamblea General de la Organización de las Naciones Unidas titulada «El derecho al desarrollo» (A/RES/54/175), adoptada en diciembre de 1986, señala en su artículo 12:

En la total realización del derecho al desarrollo, entre otros: a) El derecho a la alimentación y al consumo de agua pura son derechos humanos fundamentales, y su promoción constituye un imperativo moral tanto para los gobiernos nacionales como para la comunidad internacional.

De la voluminosa producción de instrumentos internacionales, el documento más acabado sobre el reconocimiento del derecho humano al agua y al saneamiento es la Observación General número 15 (OG15) titulada «El derecho al agua», que fue elaborada por el Consejo Económico y Social de Naciones Unidas y sancionada en noviembre de 2002. En este documento se interpretaron, por un lado, los artículos 11 y 12 del Pacto Internacional de Derechos Económicos, Sociales y Culturales y se reafirmó el derecho al agua en el ámbito internacional; ${ }^{2}$ por otro lado, se desarrollaron las bases para la implementación progresiva del derecho humano al agua, enmarcándolo en dos preceptos normativos: el derecho a un nivel de vida adecuado (artículo 11) y el derecho a disfrutar del más alto nivel de salud posible (artículo 12) (JacoboMarín, 2012).

La Observación General número 15 establece las obligaciones de los Estados en materia de derecho humano al agua y al saneamiento y define qué acciones podrían ser consideradas como una violación del mismo; el documento las relaciona no solo con el derecho a un nivel de vida adecuado y al derecho a la salud, su artículo I.1 estipula que «el derecho humano al agua es indispensable para vivir dignamente y es condición previa para la realización de otros derechos humanos» (ONU, 2002: 1).

En ese marco de enunciación política, la Resolución 64/292 denominada «El derecho humano al agua y al saneamiento» fue aprobada el 28 de julio de 2010 por la Asamblea General de las Naciones Unidas. Reconoce que «el derecho al agua potable y al saneamiento es un derecho humano esencial para el pleno disfrute de la vida y de todos los derechos humanos» (ONU, 2010: 3). Su aprobación se inscribió en los

2. El Pacto Internacional de Derechos Económicos, Sociales y Culturales fue aprobado el 16 de diciembre de 1966 y entró en vigor en México el 12 de mayo de 1981. De conformidad con el artículo 133 constitucional, los tratados celebrados por el Presidente de la República, con aprobación del Senado, forman parte del derecho interno. 
términos de los instrumentos internacionales vinculantes y no vinculantes, emitidos desde la década de los sesenta (Howard y Bartram, 2003; Jacobo-Marín, 2013).

\section{Regulación del derecho humano al agua y al saneamiento en México}

El reconocimiento explícito del derecho humano al agua y al saneamiento en el régimen jurídico mexicano se consolidó el 8 de febrero de 2012, mediante la reforma al artículo 4 de la Constitución Política de los Estados Unidos Mexicanos ${ }^{3}$, aprobada por ambas cámaras del Congreso de la Unión y las legislaturas de los estados. La reforma incorporó al mencionado artículo el siguiente párrafo:

Toda persona tiene derecho al acceso, disposición y saneamiento de agua para consumo personal y doméstico en forma suficiente, salubre, aceptable y asequible. El Estado garantizará este derecho y la ley definirá las bases, apoyos y modalidades para el acceso y uso equitativo y sustentable de los recursos hídricos, estableciendo la participación de la Federación, las entidades federativas y los municipios, así como la participación de la ciudadanía para la consecución de dichos fines. ${ }^{4}$

Adicionalmente, el artículo 115 constitucional regula los servicios públicos de abasto de agua potable, alcantarillado, drenaje, tratamiento y disposición de aguas residuales. Los gobiernos municipales en México se consideran depositarios de dichas funciones desde la publicación de las reformas constitucionales del 2 de febrero de 1983 y del 23 de diciembre de 1999. Aunque el reconocimiento del derecho humano al agua y al saneamiento en el régimen constitucional supone un avance legal, las estadísticas oficiales muestran un panorama poco alentador.

La Comisión Nacional del Agua considera que la cobertura de agua potable incluye a las personas que tienen agua entubada dentro de la vivienda; fuera de la vivienda, pero dentro del terreno; de la llave pública; o bien, de otra vivienda. Sostiene que los habitantes con cobertura no necesariamente disponen de agua con calidad para consumo humano. Considerando lo anterior, ese organismo público indica que en el año 2010 el 90,9\% de la población mexicana tenía cobertura de agua potable; para el 2015 estimó que el $96 \%$ de la población nacional (96\% urbana y 92\% rural) tenía acceso a fuentes mejoradas de agua potable (Conagua, 2016).

Lo anterior es relevante si se considera que investigaciones científicas revelan que las enfermedades asociadas al agua contaminada son la causa de millones de muertes cada año, principalmente en niños con familias de ingresos bajos y medios (OPS,

3. Diario Oficial de la Federación, «Decreto por el que se reforma y adiciona el artículo 40. de la Constitución Política de los Estados Unidos Mexicanos», 7 de mayo de 2020, disponible en https://bit. ly/3h2qFuL.

4. De acuerdo con la reforma, el Congreso de la Unión disponía de 360 días para emitir la Ley General de Aguas. Hasta el momento no ha sido aprobada. 
2012). La Comisión Nacional del Agua define el saneamiento como «la recogida y transporte del agua residual y el tratamiento tanto de esta como de los subproductos generados en el curso de esas actividades, de forma que su evacuación produzca el mínimo impacto en el medio ambiente» (Conagua, 2014: 233). La definición anterior se queda en el campo del desalojo y, en el mejor de los casos, de la remoción de algunos contaminantes en el agua, teniendo un sentido de recuperación de los costos a partir de las llamadas cuotas de saneamiento, lo que contrasta con la perspectiva de que es un asunto de calidad de vida y bienestar social, incluso con los componentes de la denominada protección ambiental.

Ahora bien, en ninguna de las regulaciones constitucionales o reglamentarias mexicanas se menciona de manera específica a los contaminantes emergentes, que se caracterizan por una alta persistencia y baja degradación en el ambiente (Tadeo y otros, 2012). Lo que se suma a que «los sistemas de tratamiento de aguas residuales que operan en México fueron diseñados para depurar sólidos, materia orgánica disuelta y nutrientes, por lo que muchos compuestos tóxicos pasan el proceso de tratamiento con poca o ninguna modificación en su concentración» (Robledo Zacarías y otros, 2017: 223). Lo mismo se puede decir de las plantas de potabilización.

\section{Los contaminantes emergentes a la luz del principio precautorio}

El inventario de contaminantes emergentes incluye una amplia variedad de productos de uso diario con aplicaciones industriales y domésticas. Algunos de ellos han sido incluidos en la lista de sustancias prioritarias en el agua de la Directiva Marco Europea; es el caso de los retardantes de llama éteres difenilos polibromados (PBDE), los detergentes de tipo alquilfenol etoxilado y las parafinas cloradas. Otras sustancias, como los pesticidas, estaban ya sujetas al control y monitoreo en la legislación sobre la calidad del agua, pero el descubrimiento de productos tóxicos de degradación ha renovado el interés por las mismas. Los retardantes de llama bromados, los detergentes de tipo alquilfenol etoxilado, los estrógenos y otros fármacos son disruptores endocrinos, lo que implica que una exposición a los mismos puede dar lugar a alteraciones en el crecimiento, desarrollo, reproducción y comportamiento de los organismos vivos. Una de las más documentadas es la feminización en organismos acuáticos superiores (Barceló y López de Alda, 2007: 12).

Los contaminantes emergentes comprenden una extensa gama de compuestos químicos, productos farmacéuticos, de cuidado personal, agentes tensoactivos, plastificantes y aditivos industriales, que no se encuentran incluidos dentro de los programas de monitoreo y tratamiento de aguas, incluyendo la síntesis de nuevos compuestos químicos o cambios en el uso y disposición de los productos químicos ya existentes, de los cuales existe poca información disponible sobre los efectos que pueden generar en la salud humana y los ecosistemas (Gil y otros, 2012: 54). 
Como se muestra a lo largo de este artículo, la presencia en mayor o menor cantidad de contaminantes emergentes en el agua de consumo humano es una realidad y bastaría con invocar el principio de precaución para que las entidades gubernamentales, encargadas del ramo, asumieran las responsabilidades correspondientes. En tal sentido, la Carta Mundial de la Naturaleza (1982) precisó el principio de precaución con tres enunciados básicos: 1) evitar las actividades que puedan causar daños irreversibles a la naturaleza; 2) las actividades que puedan entrañar peligro serán precedidas por un examen a fondo; y 3 ) las actividades no se llevarán a cabo cuando no se conozcan cabalmente sus posibles efectos perjudiciales.

En el mismo sentido, la Declaración de Río sobre el Medio Ambiente y el Desarrollo (1992) señala: «cuando haya peligro de daño grave o irreversible, la falta de certeza científica absoluta no deberá utilizarse como razón para postergar la adopción de medidas [...] para impedir la degradación del medio ambiente» (principio 15).5

El Convenio de Estocolmo sobre Contaminantes Orgánicos Persistentes (CE$\mathrm{COP}$ ) (2001) asume el criterio de precaución como base para la protección del medio ambiente y la salud humana. Este instrumento internacional fue ratificado por el Estado mexicano y, por lo tanto, forma parte del bloque de convencionalidad de acuerdo con la reforma constitucional en derechos humanos de 6 de junio de 2011, por lo que constituye un ordenamiento vinculante que debe emplearse para salvaguardar los derechos ambientales y los derechos de la naturaleza. Adicionalmente, el artículo 15 del Convenio 169 de la Organización Internacional del Trabajo (OIT) sobre Pueblos Indígenas y Tribales estipula la especial protección de los recursos naturales existentes en tierras de los pueblos originarios. ${ }^{6}$

Aunque se han desarrollado y aplicado importantes metodologías de detección (medición), así como de degradación y remoción de contaminantes emergentes, ese término es genérico e incluye una enorme cantidad de sustancias. Por ese motivo, bajo la idea de la regulación de dichas sustancias en el agua en México, en términos del principio precautorio, son aplicables los criterios vinculantes y orientadores del Convenio 169 de la OIT, el Convenio de Estocolmo sobre Contaminantes Orgánicos Persistentes y la Declaración de Río sobre el Medio Ambiente y el Desarrollo. ${ }^{7}$

5. A diferencia del principio de prevención, el de precaución se apoya en la cautela ante la falta de certeza científica y exige tomar medidas que reduzcan la posibilidad de sufrir daños ambientales, aunque se ignore la probabilidad de que estos ocurran; en tanto que el de prevención obliga a tomar medidas dado que se conocen los daños que pueden producirse.

6. El Convenio 169 de la OIT sobre Pueblos Indígenas y Tribales fue ratificado por el Estado mexicano el 13 de agosto de 1990.

7. En México, se han desarrollado interpretaciones doctrinales sobre la aplicación del principio precautorio en el contexto de la reforma constitucional en derechos humanos de 2011, de forma particular, la que se asocia a dos elementos discutidos en este apartado: la protección del medio ambiente y la salud 


\section{Contexto sociolegal del caso mexicano}

Diversos estudios han demostrado la existencia de contaminantes emergentes en cuerpos de agua en México, por ejemplo, se documenta la presencia de ibuprofeno, ketoprofeno y naproxeno en dos de los principales ríos de la ciudad Tapachula, Chiapas; también se muestra a partir de la evaluación del riesgo ambiental, utilizando cocientes de riesgo, que la presencia de contaminantes emergentes específicos presenta un posible riesgo para los organismos acuáticos (Cruz-Esteban y otros, 2014).

En el efluente de la planta de tratamiento de aguas residuales de la ciudad de Morelia, Michoacán, se identificó la presencia de tetraciclina, cefaclor, cefadroxilo, ampicilina, clonazepam, lormetazepam, secobarbital, maprotilina, levotiroxina, cisandrosterona, paracetamol, lidocaína, bromfeniramina, fexofenadina, amfetamina, morfina, benzoilecgonina, 11-nor- $\Delta 9$-THC-9-COOH, dimetilamfetamina, fenciclidina, metadona y polietilenglicol (Robledo-Zacarías y otros, 2016). En el río Nexapa se encontraron contaminantes emergentes derivados de productos del cuidado personal, aseo y componentes de los alimentos (Navarro y otros, 2014).

Algunos contaminantes orgánicos persistentes son considerados contaminantes emergentes y pueden representar un riesgo para la salud y el ambiente (Rocha-Gutiérrez, Peralta-Pérez y Zavala-Díaz, 2015). Esos mismos autores señalan que es complicado predecir el efecto de estos contaminantes, además de que están presentes en concentraciones muy bajas, en el orden de partes por trillón, pero que son bioacumulables y biomagnificables. Lo anterior implica que la aplicación del principio de precaución debe ir de la mano con el desarrollo de técnicas de detección de la presencia de contaminantes emergentes en general, pero también de las técnicas de degradación y remoción respectivas, de manera que no impacten en la salud humana $\mathrm{y}$ ambiental.

En ese sentido, de acuerdo con la hipótesis formulada en este trabajo, se argumenta que para el caso de contaminantes emergentes en México, la aplicación del principio precautorio y del marco constitucional sobre el derecho humano al agua se mantiene como una meta sociojurídica de difícil consecución, por dos motivos principales: primero, la ausencia de disposiciones normativas que regulen las referidas sustancias y que puntualicen los métodos de identificación y de remoción en aguas residuales; y, segundo, aunque los tribunales federales en México se han pronunciado sobre demandas de protección constitucional (juicio de amparo) relativas al derecho humano al agua y al saneamiento, y los criterios jurisdiccionales se refieren a elementos relevantes sobre esta prerrogativa, ${ }^{8}$ estos últimos se enfocan, fundamentalmente,

(véase Anglés, 2018) y la aplicación del Convenio 169 de la OIT sobre Pueblos Indígenas y Tribales (véase Castillo Lara, 2017).

8. Véase Semanario Judicial de la Federación, 2018, 2017, 2016 y 2015. 
en la asequibilidad del servicio, la suspensión del suministro y la continuidad del abasto, cuestiones que se han discutido al margen de la calidad del agua para el consumo humano.

En ese orden de ideas, es relevante considerar que el vacío legal sobre contaminantes emergentes en México se manifiesta como una arista pendiente en el contexto del cumplimiento progresivo del derecho humano al agua y al saneamiento, sobre todo, porque el saneamiento adecuado y el suministro de agua de calidad son actividades cuya observancia compete a las autoridades de los tres órdenes de gobierno, de acuerdo con el marco de gestión y distribución de competencias establecidos en los artículos 4, 27 y 115 de la Constitución.

\section{Regulación jurídica de los contaminantes emergentes en México}

Los parámetros y valores característicos de contaminantes que se presentan en el agua se desarrollaron en México, para fines de regulación oficial, por primera vez en 1976, mediante los estudios elaborados por la Secretaría de Recursos Hidráulicos. Esta entidad perfiló los contaminantes típicos que se esperaban en las aguas residuales de cinco industrias (alimenticia, textil, curtiduría, química y acabado de metales). Dichos estudios revelan la dificultad de evaluar el grado de contaminación en las aguas residuales de origen industrial y la necesidad de establecer límites para estimar el consumo de agua y la generación de aguas residuales por unidad de producto terminado, o bien, por unidad de materia prima transformada (Ramírez Cortina, 1992: 13).

En este contexto, los criterios se regularon en las Normas Técnicas Ecológicas para el Control de la Calidad del Agua que se publicaron en el Diario Oficial de la Federación el 4 de agosto de $1988,{ }^{9}$ y los Criterios Ecológicos de Calidad del Agua, que se emitieron el 13 de diciembre de $1989^{10}$. Las Normas Técnicas Ecológicas establecían los límites máximos permisibles de los contaminantes específicos en las descargas de aguas residuales de 30 sectores industriales; se expidieron 25 de estas normas con el propósito de reglamentar la calidad del agua residual vertida en cuerpos receptores, como ríos, cauces, vasos, aguas marinas y demás depósitos o corrientes de agua. ${ }^{11} \mathrm{Di}$ -

9. Véase https://bit.ly/3h1lBqA.

10. Véase https://bit.ly/3x6GFS5.

11. Las industrias reguladas eran las siguientes: 1) centrales termoeléctricas convencionales; 2) producción de azúcar de caña; 3) refinación de petróleo crudo, sus derivados y petroquímica básica; 4) fabricación de fertilizantes (excepto las que producían ácido fosfórico como producto intermedio); 5) plásticos y polímeros sintéticos; 6) fabricación de harinas; 7) cerveza y malta; 8) fabricación de asbestos de construcción; 9) elaboración de leche y sus derivados; 10) manufactura de vidrio plano; 11) producción de vidrio prensado y soplado; 12) fabricación de caucho sintético, llantas y cámaras; 13) hierro y acero; 14) textil, 15) celulosa y papel; 16) bebidas gaseosas; 17) acabados metálicos; 18) laminación, extrusión 
cha normativa también sugería los tipos de tratamiento que podrían aplicarse a las descargas para no rebasar los límites máximos permitidos (SARH, 1976). ${ }^{12}$

Los límites máximos permisibles de contaminantes se fijaron en parámetros de acuerdo con el promedio diario y el valor instantáneo. El promedio diario se calcula mediante el análisis de muestras compuestas que resultan de la mezcla de muestras instantáneas tomadas a intervalos durante el proceso generador de la descarga.

Las Normas Técnicas Ecológicas eran emitidas por la Secretaría de Desarrollo Urbano y Ecología con la finalidad de establecer los requisitos, especificaciones, condiciones, procedimientos, parámetros y límites permisibles que debían observarse en el desarrollo de las actividades, uso y destino de bienes naturales, que pudieran causar un desequilibrio ecológico o daño ambiental. La publicación de las Normas Técnicas Ecológicas comenzó el 6 de junio de 1988 y, tres meses después, entró en vigor la Ley General del Equilibrio Ecológico y la Protección al Ambiente. Desde la perspectiva oficial, las normas tenían como finalidad unificar los criterios, principios y políticas en materia ambiental, «determinando estándares de emisión para garantizar las condiciones necesarias para el bienestar de la población, y asegurar la preservación y restauración del equilibrio ecológico» (Ramírez Cortina, 1992: 25).

Ahora bien, las Normas Técnicas Ecológicas sobre el Control de la Contaminación del Agua constituyen el antecedente jurídico de las actuales Normas Oficiales Mexicanas sobre calidad, descarga y tratamiento del agua. ${ }^{13}$ En términos del objetivo de este trabajo, es relevante señalar que existe un entramado de regulación relativo a los límites máximos permisibles de contaminantes en las descargas de aguas residuales, ${ }^{14}$ sobre los límites permisibles de calidad del agua para el consumo humano ${ }^{15} \mathrm{y}$ sobre

y estiraje de cobre y sus aleaciones; 19) impregnación de productos de aserradero; 20) asbestos textiles, materiales de fricción y selladores; 21) curtido y acabado de pieles; 22) matanza de animales y empacado de cárnicos; 23) envasado de conservas alimenticias; 24) elaboración de papel a partir de celulosa virgen; y 25) elaboración de papel a partir de fibra celulósica reciclada.

12. Los estudios de calidad del agua fueron realizados por la Secretaría de Recursos Hidráulicos (SRH), disuelta el 30 de noviembre de 1976 durante el gobierno de José López Portillo. La SRH fue suplida por la Secretaría de Agricultura y Recursos Hidráulicos (SARH), en el marco de gestión y regulación contenido en la Ley Federal de Aguas de 1972.

13. Hasta octubre de 1991 se publicaron 31 Normas Técnicas Ecológicas sobre el Control de la Contaminación del Agua en el Diario Oficial de la Federación.

14. Los límites máximos permitidos de contaminantes en las descargas de aguas residuales de propiedad nacional en los sistemas de alcantarillado urbano o municipal y para las aguas residuales tratadas que se reúsan en servicios al público están regulados en las Normas Oficiales Mexicanas NOM-oo1-SEMARNAT-1996, NOM-o02-ECOL-1996 y NOM-o03-ECOL-1997. La referida normativa se publicó en el Diario Oficial de la Federación el 6 de enero de 1997, el 3 de junio de 1998 y el 21 de septiembre de 1998, respectivamente.

15. La NOM-127-SSA1-1994 establece los límites permisibles de calidad y tratamientos a que debe someterse el agua para su potabilización, para uso y consumo humano. La modificación más reciente a dicha norma fue publicada en el Diario Oficial de la Federación el 22 de noviembre de 2000. 
buenas prácticas para la fabricación de medicamentos. ${ }^{16}$ Sin embargo, el análisis detallado de su contenido revela un vacío legal sobre los contaminantes emergentes en el agua, es decir, de acuerdo con lo expuesto por Daughton (2004), podría hablarse propiamente de «contaminantes no regulados».

Ciertos contaminantes pueden emplearse como herramientas trazadoras que proporcionan una indicación inmediata sobre la presencia de otra clase de contaminantes que, dada su naturaleza, permanencia temporal en el agua y costos de identificación, son difíciles de medir. Por ejemplo, los $\mathrm{PPCP}^{17}$ pueden ser usados como indicadores de intrusión de aguas residuales (rastreo de columnas de aguas residuales o determinación de la fuente de contaminación del agua subterránea). El monitoreo de PPCP también puede emplearse para documentar el comportamiento adverso de contaminantes emergentes en el agua derivado del uso de drogas ilícitas o sustancias reguladas usadas discrecionalmente (Daughton 2004).

No obstante, la regulación jurídica de los contaminantes emergentes requiere una amplia investigación sobre sus efectos potenciales en la salud (USEPA, 2014; USGS, 2014), es decir, de estudios de toxicidad, toda vez que «la escasez de datos ambientales disponibles sobre los contaminantes emergentes dificulta su regulación a través de las normas mexicanas para el control de la polución del agua» (Robledo-Zacarías y otros, 2017: 223), además de que la evaluación del impacto y la regulación de estos compuestos en el medio ambiente requiere de métodos analíticos con límites de detección suficientemente bajos (Daughton 2004; Tadeo y otros, 2012).

Para el caso mexicano, se ha documentado la presencia (tipos y concentraciones) de contaminantes emergentes en diversas zonas del país; además se están investigando los posibles efectos en la salud humana y ambiental. Esta información es muy útil si se encamina a su regulación normativa; sobre todo porque la presencia de los «contaminantes no regulados» plantea desafíos sustanciales y quizá insuperables para su regulación y control (Becerril, 2009), toda vez que se tiene un alto desconocimiento de sus efectos y no existe un inventario de todas las especies químicas presentes en el ambiente, lo que se asocia con limitaciones analíticas (Becerril, 2009; Navarro y otros, 2014).

Tradicionalmente se ha usado el enfoque reactivo para abordar la presencia de

16. La NOM-059-SSA1-2015 contiene los requisitos mínimos necesarios para el proceso de fabricación de los medicamentos para uso humano comercializados en el país y/o con fines de investigación. Es de observancia obligatoria para todos los establecimientos dedicados a la fabricación o importación de medicamentos para uso humano, así como para los laboratorios de control de calidad, almacenes de acondicionamiento, depósito y distribución de medicamentos. Esta norma se publicó en el Diario Oficial de la Federación el 5 de febrero de 2016.

17. La sigla PPCP (Pharmaceuticals and Personal Care Products) se emplea en la literatura sobre contaminantes emergentes y sus impactos ambientales. Los PPCP incluyen una amplia gama de sustancias utilizadas con fines cosméticos, el cuidado personal y la salud. 
contaminantes no regulados o de contaminantes conocidos que reaparecen en el agua. El hecho de que las acciones correctivas se emplearan mucho tiempo después de que los productos alcanzaran concentraciones medibles ha conducido a la discusión sobre el principio de precaución. Posteriormente, el paradigma de enfoque proactivo fue acogido con beneplácito en las investigaciones sobre contaminantes emergentes con miras a ser incorporado en las regulaciones oficiales. Este enfoque se anticipa a las contingencias generadas por la presencia de contaminantes emergentes, antes de cualquier prevención o medidas correctivas, mediante el diseño de programas que modelen escenarios adversos, por ejemplo, usando nanomateriales (Daughton, 2004).

En México, en materia de agua potable, la norma oficial mexicana NOM-127SSA1-1994 establece los límites permisibles de calidad del agua para uso y consumo humano y sus tratamientos de potabilización. Incluye para tal fin características bacteriológicas, químicas, radiactivas, físicas y organolépticas, pero no productos de tipo farmacéuticos, sin embargo, contempla que en el caso de alguna contingencia, resultado de la presencia de sustancias especificadas o no en la referida norma, se deben coordinar la autoridad sanitaria competente, las autoridades locales, la Comisión Nacional del Agua, los responsables del abastecimiento y los particulares, instituciones públicas o empresas privadas involucradas, para determinar las acciones que se deben realizar con relación al abastecimiento de agua a la población.

Por otro lado, el conocimiento científico sobre el destino y los efectos de los contaminantes emergentes en el agua ha evolucionado considerablemente a lo largo de los últimos años. Actualmente se sabe más sobre cuál es el compartimento del medio acuático (agua, sedimentos o biota) en que resulta probable encontrar una sustancia y, por lo tanto, en que es más probable que pueda medirse su concentración. Algunas sustancias muy hidrófobas se acumulan en la biota y son difícilmente detectables en el agua, incluso utilizando técnicas analíticas avanzadas.

Los contaminantes emergentes son cada vez más estudiados debido a su amplia presencia en el ambiente, además de que no son comúnmente monitoreados por la falta de regulación (Tadeo y otros, 2012). En Estados Unidos de América fue a fines de la década de los noventa cuando la Environmental Protection Agency (EPA) comenzó a documentar la presencia de contaminantes emergentes, aunque Daughton (2004) señala que es mejor denominarlos contaminantes no regulados debido a que la idea de emergentes proviene de que muy apenas, cuando menos en México, se está procediendo a su detección en algunos centros de investigación y universidades; y por esa razón no han dado pie al establecimiento de políticas públicas y regulaciones.

Ahora bien, en los marcos normativos de la Unión Europea y de Estados Unidos se han desarrollado regulaciones específicas para los llamados productos farmacéuticos, considerando su monitoreo y sus efectos en la salud humana y ambiental (Castro-Pastrana y otros, 2015). A pesar de ello, las referencias bibliográficas espe- 
cializadas señalan que solo el o,03\% de las sustancias químicas con potencial tóxico, cuyo total es de alrededor de cien millones, están reguladas (Castro-Pastrana y otros, 2015). En tal sentido, Jáuregui-Medina y otros (2015) indican que de acuerdo con las Guías para la Calidad del Agua Potable de la Organización Mundial de la Salud (OMS), no es necesario desarrollar valores de referencia formales para los productos farmacéuticos debido a que las bajas concentraciones de medicamentos que se han registrado implican un bajo riesgo para la salud humana. Así, la OMS solo considera riesgos microbianos y químicos, derivados estos últimos de la presencia de metales pesados en el agua.

\section{Perspectiva comparada con los marcos normativos sobre sustancias químicas contaminantes de la Unión Europea y Chile}

Como se ha señalado, en México no se ha desarrollado, hasta el momento, un marco regulatorio sobre la presencia de contaminantes emergentes en las fuentes de suministro de agua. En ese orden de ideas, en este apartado se examina, de forma breve, la regulación sobre la calidad de las aguas y, de forma particular, la relativa a los compuestos químicos contaminantes en los marcos normativos de Chile y de la Unión Europea. Lo anterior responde a la estrategia metodológica y la línea de análisis propuesta en este trabajo; en tal sentido, la elección de los casos persigue fines específicos. En primer lugar, se expone el marco legal comunitario de la Unión Europea, articulado en un conjunto de directivas relativas a la política de aguas, porque se trata del corpus normativo más detallado sobre sustancias contaminantes emergentes en el agua, de manera que es viable comparar este esquema con los órdenes jurídicos de México y de Chile.

En segundo lugar, se muestra el estado de reglamentación sobre las referidas sustancias en el ámbito jurídico de América Latina, a partir de la revisión del caso chileno, cuya característica distintiva es su modelo sui generis de gestión, control y asignación de las aguas. Se considera que el esquema de gestión hídrica en Chile es una característica relevante en el contexto de regulación de la calidad de las aguas y del derecho humano al agua, porque se ha documentado en la literatura el modelo neoliberal de asignación de los derechos de agua y su relación con el surgimiento de diversos conflictos en el país. ${ }^{18}$

Esta revisión comparativa permite mostrar el vacío normativo sobre contaminantes emergentes en el agua en México y Chile.

18. Sobre este argumento, consúltense los trabajos de Guerrero-Valdebenito y otros (2018), Castro y Quiroz (2011) y Larraín (2006). Desde una óptica distinta, se ha defendido el modelo de derechos de agua asignados por el mercado, véase, por ejemplo, el trabajo de Matthews (2004). 


\section{Unión Europea}

En el marco común europeo, un conjunto de directivas permite analizar la regulación sobre los contaminantes emergentes en el agua. Para ello, los Estados miembros de la Unión Europea han convenido un esquema de competencias basado en los principios de atribución, subsidiariedad y proporcionalidad. El Tratado de Funcionamiento de la Unión Europea (TFUE) establece la competencia compartida con los Estados miembros en materia de medio ambiente (artículo 4, apartado 2, inciso e) ${ }^{19}$. La facultad para legislar en dicha materia está regulada por el artículo 191, cuya base procedimental se funda en los principios de cautela y de acción preventiva, en el principio de corrección de los atentados al medio ambiente y en el principio de quien contamina paga.

La Unión Europea ha sugerido que la armonización normativa necesaria para la protección ambiental debe incluir una cláusula de salvaguardia que autorice a los Estados miembros a adoptar, por motivos medioambientales no económicos, medidas provisionales sometidas a un procedimiento de control. ${ }^{20}$ La delimitación de competencias que se ubica en el artículo 5 del Tratado de la Unión Europea (TUE) se rige por el principio de atribución, y el ejercicio de las competencias se rige por los principios de subsidiariedad y de proporcionalidad. ${ }^{21} \mathrm{El}$ principio de atribución indica que la Unión Europea actúa dentro de los límites de las competencias que le atribuyen los Estados miembros para lograr lo establecido en los tratados. Mediante el principio de subsidiaridad, en los ámbitos que no sean de su competencia exclusiva, la comunidad interviene solo en caso de que los objetivos de la acción pretendida no puedan ser alcanzados por los miembros a escala local o regional. ${ }^{22}$ En virtud del principio de proporcionalidad, el contenido y la forma de la acción comunitaria no excederán de lo necesario para alcanzar los objetivos de los tratados.

La Directiva 2011/92/UE establece los principios generales de evaluación de impacto ambiental de los proyectos públicos y privados que puedan generar repercusiones negativas, en particular sobre la calidad de las aguas. Esta directiva recomienda la fijación de criterios para estudiar los proyectos caso por caso, con la finalidad de determinar cuáles han de someterse a una evaluación en función de la relevancia de

19. https://eur-lex.europa.eu/legal-content/ES/TXT/PDF/?uri=CELEX:12016E/TXT\&from=ES

20. Dicho reconocimiento se realizó en la modificación del año 2010 del Tratado de Funcionamiento de la Unión Europea, disponible en https://bit.ly/3w6Itcq.

21. Véase https://bit.ly/3w2UDDo.

22. Las instituciones de la Unión Europea aplican el principio de subsidiariedad de conformidad con el Protocolo sobre la Aplicación de los Principios de Subsidiariedad y Proporcionalidad. Los parlamentos nacionales velan por el respeto del principio de subsidiariedad con arreglo al procedimiento establecido en el mencionado protocolo. 
sus repercusiones, de conformidad con el principio de subsidiariedad. ${ }^{23}$ La Directiva 2011/92/UE también describe los proyectos que han de someterse a evaluación de impacto ambiental, incluyendo las plantas de tratamiento de aguas residuales urbanas (anexo 1, artículo 13) y la estimación de los tipos, cantidades y emisiones de residuos que puedan contaminar el agua (anexo 4, artículo 1, inciso c), que circunscriben, de conformidad con el anexo 2, a la agricultura, la industria extractiva, las instalaciones industriales para la producción de electricidad, la producción y elaboración de metales, las industrias de minerales, química, alimenticia, textil, cuero, madera, papel, caucho y otros proyectos de infraestructura, incluido el turismo.

En el mismo sentido, la Directiva 2006/21/CE establece medidas, procedimientos y orientaciones para prevenir los efectos adversos sobre las aguas y la salud humana derivados de la gestión de los residuos de las industrias extractivas. En este contexto, los Estados miembros de la Unión Europea deben obligar a las entidades que participan en las industrias extractivas a aplicar las mejores técnicas disponibles de seguimiento y control para prevenir la contaminación del agua y el suelo, e identificar cualquier efecto adverso que las instalaciones de residuos puedan tener sobre el medio ambiente y la salud de las personas. ${ }^{24}$ Por su parte, la Directiva 2000/60/CE, del 23 de octubre de 2000 , regula la actuación comunitaria en el ámbito de la política de aguas. Esta directiva establece un marco para la protección de las aguas superficiales continentales, las aguas de transición, las aguas costeras y las aguas subterráneas, para prevenir su deterioro y garantizar la reducción progresiva de los vertidos, las emisiones y las sustancias peligrosas prioritarias ${ }^{25}$. De forma específica, la Directiva 2000/60/CE indica la formulación de programas en cada Estado que contengan medidas en los planes hidrológicos de cuenca (artículo 11, inciso j). Se estableció, también, la prohibición de vertidos directos de contaminantes en las aguas subterráneas. Para el caso concreto de contaminantes emergentes, los Estados miembros aceptaron emitir Normas de Calidad Ambiental para otra matriz o, en su caso, otros taxones de la biota, por ejemplo, el subfilum Crustacea, parafilum (peces), clase Cephalopoda o clase Bivalvia (mejillones y almejas) ${ }^{26}$.

La Directiva 2013/39/UE reconoce que la contaminación química de las aguas superficiales y subterráneas representa una amenaza para el medio acuático, con efectos

23. Directiva 2011/92/UE, Evaluación de las repercusiones de los proyectos sobre el medio ambiente (evaluación de impacto ambiental), disponible en https://bit.ly/3hkgluT.

24. Directiva 2006/21/CE, sobre la gestión de los residuos de industrias extractivas y por la que se modifica la Directiva2004/35/CE, disponible en https://bit.ly/2UIvGzT.

25. Directiva 2000/60/CE, por la que se establece un marco comunitario de actuación en el ámbito de la política de aguas, disponible en https://bit.ly/3qvNutS.

26. DIRECTIVA 2013/39/UE, por la que se modifican las Directivas 2000/60/CE y 2008/105/CE en cuanto a las sustancias prioritarias en el ámbito de la política de aguas, disponible en https://bit. ly/3joQuGY. 
como la toxicidad aguda y crónica en organismos acuáticos, acumulación de contaminantes en el ecosistema y pérdida de hábitats y de biodiversidad, lo que también supone una amenaza potencial para la salud humana. Los anexos 1 y 2 de esta directiva listan las sustancias prioritarias en el ámbito de la política de aguas de la Unión Europea. Sin tratarse de un listado exhaustivo, es el instrumento más acabado en términos de regulación de las sustancias identificadas, convencional y jurídicamente, como «peligrosas».

Finalmente, de acuerdo con la revisión del marco común europeo sobre la política de aguas, se observa que se trata del corpus normativo más detallado sobre sustancias contaminantes emergentes. Adicionalmente, de acuerdo con los principios para la delimitación de competencias, se ha sugerido, en el ámbito comunitario, que cada Estado adopte medidas específicas para la regulación de tales compuestos químicos, en el contexto de la armonización normativa para la protección de la salud humana $\mathrm{y}$ ambiental.

\section{Chile}

Con la promulgación de la Constitución Política de 1980 y la dictación del Código de Aguas en 1981, se introdujeron modificaciones sustanciales en el derecho chileno de aguas. Los títulos se otorgan a través de un acto administrativo que incorpora al patrimonio o dominio de su titular el uso, goce y disposición de conformidad con la ley. La noción de propiedad individual sobre el agua, separada de la tierra, permite al titular de un derecho comercializar con este (enajenar, hipotecar, arrendar), de manera que se ha desarrollado un «mercado de agua» donde se hayan inscritos los aprovechamientos hidráulicos (Larraín, 2006).

Para el caso de la calidad de las aguas, el artículo 129 bis 3 del Código de Aguas establece que la Dirección General de Aguas deberá establecer una red de estaciones de control de calidad, cantidad y niveles de las aguas tanto superficiales como subterráneas en cada cuenca u hoya hidrográfica. La información que se obtenga deberá ser pública y proporcionarse a quien la solicite.

Por otro lado, la Norma Chilena Oficial NCh1333 establece los requisitos de calidad para diferentes usos, de acuerdo con requerimientos referidos a aspectos físicos, químicos y biológicos, según el uso determinado. Conforme lo establecido por la referida norma, el objetivo es proteger y preservar la calidad de las aguas de la degradación producida por contaminación con residuos de cualquier tipo u origen. La NCh1333 se aplica a las aguas destinadas al consumo humano, la bebida de animales, el riego, la recreación y estética (con y sin contacto directo) y la vida acuática. Sin embargo, dicha norma no se pronuncia respecto de contaminantes emergentes y solo refiere que el vaciamiento de residuos contaminantes a masas o cursos de agua deberá ajustarse a los requerimientos de calidad especificados para cada uso, teniendo en 
cuenta la capacidad de autopurificación y dilución del cuerpo receptor, de acuerdo con los estudios que efectúe la autoridad competente en cada caso particular ${ }^{27}$.

Como sucede en México, en Chile no se ha desarrollado una regulación específica para contaminantes emergentes en el agua, no obstante que se han documentado concentraciones de hasta $20 \mu \mathrm{g} / \mathrm{l}$ de sustancias como ácido acetilsalicílico, ibuprofeno, naproxeno, ketoprofeno, ácido mefenámico, diclofenaco, ácido clorfíbrico, gemfibrozilo, parabenos, hormonas, nonilfenol y triclosán en aguas residuales y ríos de la Región Metropolitana (Maipo en este caso) y, de hasta $8 \mu \mathrm{g} / \mathrm{l}$ en aguas sometidas a procesos de remoción en las principales plantas de tratamiento (Manzo y otros, 2019).

El triclosán, por ejemplo, que se emplea en productos de aseo personal (jabones, dentífricos o talcos), está prohibido en algunos países de Europa, pero en Chile no se cuenta con una regulación específica; las moléculas de triclosán generan daños en el sistema endocrino y contaminación de diversa índole en la biota acuática. De acuerdo con la investigación de Manzo y otros (2019), la generación de contaminantes emergentes comienza en cualquier domicilio u hospital, derivado de fármacos y productos de cuidado personal regulados que luego de su uso van al sistema de alcantarillado de forma nativa o como algún metabolito (molécula producida durante el metabolismo), de modo que, luego de la ingesta de antiinflamatorios, analgésicos, antipiréticos y antidepresivos una parte inalterada es expulsada en la orina.

En la cuenca del río Biobío se ha registrado la presencia de antidepresivos, antibióticos (de uso humano y veterinario), betabloquedores, reguladores de lípidos, estimulantes y herbicidas en aguas servidas (Henríquez Villa, 2012). Lo que reconoce la literatura especializada de forma unánime es que estos compuestos tienen una alta solubilidad en el agua y una parte considerable de ellos no se remueve en las plantas de tratamiento de aguas residuales. Ahora bien, un volumen considerable de esa agua llega a los ríos o lagos a través del efluente $y$, en tal sentido, no puede descartarse la presencia de trazas de contaminantes emergentes en el agua para consumo humano donde existen tomas de agua de río que no cuentan con otras fuentes (Manzo y otros, 2019).

Lo que no se conoce con certeza son los efectos que la acumulación de dichos compuestos químicos puede causar a largo plazo en la salud ambiental y humana, lo que ha dificultado la reglamentación en el régimen jurídico de Chile. En todo caso, es preferible, en los casos mexicano y chileno, atender los postulados del principio precautorio, sobre todo porque existe suficiente documentación e instrumentos internacionales que pueden traducirse en políticas de calidad de las aguas. La idea es materializar mecanismos de protección efectivos en lugar de medidas reactivas de contingencia.

27. Norma Chilena Oficial NCh1333.Of78, 1987, disponible en https://bit.ly/3yzbbfH. 


\section{Conclusiones}

De acuerdo con el análisis elaborado en este trabajo se concluye que la presencia de contaminantes emergentes derivada de actividades antropogénicas no está siendo atendida de forma integral por la reglamentación ni por las alternativas tecnológicas para su remoción. Desde luego, la mayor parte de las investigaciones que documentan la presencia de contaminantes emergentes en los cuerpos de agua de México se enfrentan con este vacío legal y emplean las regulaciones internacionales como valores guía, pero también señalan que en un determinado cuerpo de agua pueden estar presentes un gran número de contaminantes, en cantidades variables, de manera que esto se ha interpretado como un obstáculo para su regulación jurídica.

En tal sentido, diversas investigaciones científicas demuestran la presencia de contaminantes emergentes en el territorio mexicano, cuyos orígenes son variados, como variados son sus efectos en el ambiente y la salud humana. Si bien se requieren técnicas de detección muy precisas, pero sobre todo técnicas de degradación y remoción muy efectivas, estas se están desarrollando cada vez más en los centros de investigación en México. Aunque los avances técnico-normativos son importantes, son aún escasos si se les compara con el marco normativo comunitario de la Unión Europea.

Lo mismo ocurre con los marcos regulatorios sobre agua potable/residual en México, los cuales no consideran la importancia de la detección y, aún menos, la degradación o remoción de contaminantes emergentes en agua para consumo humano. De allí la necesidad de plantear la importancia de discutir pero, más que eso, regular la presencia de esas sustancias, en todo caso, considerando los postulados formulados por el principio precautorio. No obstante la voluminosa producción de instrumentos que declaran el derecho humano al agua de calidad aceptable en el ámbito internacional, incluidas la Observación General número 15 y la Resolución 64/29, aprobadas en noviembre de 2002 y julio de 2010, respectivamente, en el ámbito orgánico de Naciones Unidas, puede concluirse que el saneamiento y el suministro de agua de calidad para el uso y el consumo humano han quedado al margen del debate sobre los contaminantes emergentes. Sobre todo, si se considera que la bibliografía especializada y los informes oficiales revelan que las enfermedades asociadas al agua contaminada son la causa de millones de muertes cada año, principalmente en niños con familias de ingresos bajos y medios.

Ahora bien, las Guías para la Calidad del Agua Potable de la Organización Mundial de la Salud (OMS) no consideran necesario desarrollar valores de referencia formales para los productos farmacéuticos debido a que las bajas concentraciones de medicamentos que se han registrado implican un bajo riesgo para la salud humana. De este modo, la OMS solo considera riesgos microbianos y químicos, derivados de la presencia de metales pesados en el agua. 
En este contexto, la ausencia de dispositivos que normen los contaminantes emergentes en México obstaculiza el cumplimiento del derecho humano al agua y al saneamiento, sobre todo el que se refiere a dos aspectos centrales: el adecuado saneamiento y la calidad del agua suministrada para el consumo doméstico. Lo anterior implica desafíos sustanciales para su regulación y control, toda vez que se tiene un alto desconocimiento de sus efectos y no existe un inventario de todas las sustancias químicas presentes en el ambiente, lo que se asocia con limitaciones analíticas.

En conclusión, puede verse que es un círculo vicioso, en el sentido de que las plantas de tratamiento de aguas residuales en México, en los lugares en los que existen y operan con eficiencia, no están diseñadas para remover los contaminantes emergentes (García, Gortáres y Drogui, 2011; Robledo-Zacarías y otros, 2017), pero ello también ocurre en países desarrollados como Estados Unidos de América (Dougherty y otros, 2010), y sus efluentes pueden contaminar, a su vez, cuerpos de agua (ríos, acuíferos, zonas marinas, etcétera) que posteriormente se convierten en fuente de suministro del líquido para las plantas potabilizadores que, igualmente, no están diseñadas para la remoción o degradación de estos contaminantes. En tal sentido, debe reconocerse que la discusión regulatoria y normativa debería considerar, tomando en cuenta lo señalado por Daughton (2004) y Dougherty y otros (2010), aspectos como la clase de contaminantes emergentes en relación con su estructura química, el uso, el tipo de efectos, sus mecanismos de acción, las fuentes y las rutas de exposición, entre otros.

Finalmente, respecto de la revisión comparativa, se concluye que hasta el momento no se han desarrollado normativas específicas sobre la regulación de contaminantes emergentes en el agua en México y Chile. Aunque en los dos países se cuenta con un corpus nutrido sobre los derechos de agua y la calidad del líquido para el uso y el consumo humano, es evidente el vacío normativo sobre el tópico que se analiza en este artículo. Esta ausencia se hace patente de forma más amplia si se le compara con el marco comunitario de la Unión Europea, en cuyo ámbito, la Directiva 2013/39/UE contiene un listado detallado de sustancias identificadas, de forma técnica y jurídica, como «peligrosas».

\section{Referencias}

ANGLÉs, Marisol (2018). «El principio precautorio en México: plaguicidas, medio ambiente y salud». En S. Chan, F. Ibarra y M. J. Medina (editores), Bioética y bioderecho. Reflexiones clásicas y nuevos desafíos (pp. 439-459). México: Instituto de Investigaciones Jurídicas-UNAM. Disponible en https://bit.ly/3diZCob.

BAI, Xuelian, Alex Lutz, Rosemary Carroll,, Kristen Keteles, Kenneth Dahlin, Mark Murphy y David Nguyen (2018). «Occurrence, distribution, and seasonality of emerging contaminants in urban watersheds». Chemosphere, 200: 133-142. DOI: 10.1016/j.chemosphere.2018.02.106. 
BARCeló, Damià y María José López de Alda (2007). «Contaminación y calidad química del agua: el problema de los contaminantes emergentes». En Panel Científico-Técnico de Seguimiento a la Política de Aguas. Zaragoza: Fundación Nueva Cultura del Agua.

BeCerril, José (2009). «Contaminantes emergentes en el agua». Revista Digital Universitaria, 8 (10): 2-7. Disponible https://bit.ly/3d3u89Y.

Castillo Lara, Clara (2017). «La Constitución mexicana y el Convenio 169 de la OIT sobre pueblos indígenas y tribales». Alegatos, 97: 559-578. Disponible en https://bit.ly/2TUEYIU.

CAStro, Milka y Loreto Quiroz (2011). «La crisis del agua en Chile: el futuro de Chile requiere una nueva política de agua». En R. Boelens, L. Cremers y M. Zwarteveen (editores), Justicia hídrica. Acumulación, conflicto y acción social (pp. 225-240). Lima: Pontificia Universidad Católica del Perú, Instituto de Estudios Peruanos, Alianza Internacional Justicia Hídrica.

Castro-Pastrana, Lucila, María Baños-Medina, María López-Luna y Blanca Torres-García (2015). «Ecofarmacovigilancia en México: Perspectivas para su implementación». Revista Mexicana de Ciencias Farmacéuticas, 46 (3): 16-40. Disponible en https://bit.ly/2TW8LAH.

Conagua, Comisión Nacional del Agua (2014). Estadísticas del agua en México. México: Secretaría de Medio Ambiente y Recursos Naturales. Disponible en https:// bit.ly/3jo YyoE.

-. (2016). Estadísticas del agua en México. México: Secretaría de Medio Ambiente y Recursos Naturales. Disponible en https://bit.ly/3vPtjYW.

Cruz-Esteban, Samuel, Leopoldo Cruz-López, Edi Malo, Javier Valle-Mora, Dulce Infante-Matha, Antonia Santiesteban-Hernández, Rubén Gutiérrez Hernández, y Ricardo Bello-Mendoza (2014). «Presencia de anti-inflamatorios no esteroideos en cuerpos de agua superficial de Tapachula, Chiapas, México». Revista AIDIS de Ingeniería y Ciencias Ambientales, 7 (2): 105-114. Disponible en http://dx.doi. org/10.22201/iingen.0718378xe.2014.7.2.46788

Daughton, Christian G. (2004). «Non-regulated water contaminants: emerging research». Environmental Impact Assessment Review, 24 (7-8): 711-732. DOI: 10.1016/j.eiar.2004.06.003.

Dougherty, Jennifer A., Peter W. Swarzenski, Richard S. Dinicola, y Martin Reinhard (2010). «Occurrence of herbicides and pharmaceutical and personal care products in surface water and groundwater around Liberty Bay, Puget Sound, Washington». Journal of Environmental Quality, 39 (4): 1.173-1.180. DOI: 10.2134/ jeq2009.0189.

García, Celestino, Pablo Gortáres y Patrick Drogui (2011). «Contaminantes emergentes: efectos y tratamientos de remoción». Química Viva, 10 (2): 96-105. Disponible en https://www.redalyc.org/pdf/863/86319141004.pdf. 
GIL, Miriam, Adriana Soto, Jorge Usma y Omar Gutiérrez (2012). «Contaminantes emergentes en aguas, efectos y posibles tratamientos». Producción + Limpia, 7 (2): 52-73. Disponible en https://bit.ly/3h8dzv5.

Guerrero-Valdebenito, Rosa María, Francisca Fonseca-Prieto, Jaime GarridoCastillo y Mauricio García-Ojeda (2018). «El código de aguas del modelo neoliberal y conflictos sociales por agua en Chile: Relaciones, cambios y desafíos». Agua y Territorio, 11: 97-108. DOI: 10.17561/at.11.3956.

Henríquez Villa, Deyanira (2012). Presencia de contaminantes emergentes en aguas y su impacto en el ecosistema. Estudio de caso: productos farmacéuticos en la cuenca del río Biobío, región del Biobío, Chile. Tesis de Magíster. Universidad de Chile.

Howard, Guy y Jamie Bartram (2003). Domestic water quantity, service level and health. Ginebra: Organización Mundial de la Salud.

JACOBO-Marín, Daniel (2012). «El acceso al agua en México: ¿un derecho humano?». En D. Cienfuegos y G. Cordero (editores), Estudios sobre derechos individuales $y$ de grupo (pp. 137-154). México: Instituto de Investigaciones Jurídicas, UNAM. DOI: 10.13140/RG.2.1.3166.6087.

Jacobo-Marín, Daniel (2013). «Agua para San Luis Potosí: Una mirada desde el derecho humano al agua en dos sectores del ámbito urbano». Tesis de Maestría. El Colegio de San Luis. DOI: 10.13140/RG.2.1.2970.0009/1.

JÁuregui-Medina, Cecilia, Lesset Ramos-Ramírez, Raquel Medina Carrillo, Jorge Figueroa-Morales, Imelda Rodríguez-Castañeda y Roberto Padilla-Noriega. (2015). «México hacia una cultura sobre la disposición final de medicamentos caducos». Revista Fuente, 6 (20): 24-31.

LARRAÍn, Sara (2006). "El agua en Chile: entre los derechos humanos y las reglas del mercado». Polis, 14: 1-20. Disponible en https://journals.openedition.org/ polis/5091.

Manzo, Valentina, Jairón Goya-Pacheco, Daniel Arismendi, Mercedes Becerra-Herrera, Alver Castillo-Aguirre, Rosario Castillo-Felices, Milton Rosero-Moreano, Eduardo Carasek y Pablo Richter (2019). "Cork sheet as a sorptive phase to extract hormones from water by rotating-disk sorptive extraction (RDSE)». Analytica Chimica Acta, 1087 (9): 1-10. DOI: 10.1016/j.aca.2019.08.069.

Matthews, Olen P. (2004). «Fundamental questions about water rights and market reallocation». Water Resources Research, 40: 1-8. DOI: 10.1029/2003WRoo2836.

Navarro, Amado, Jorge Herrera, José Marrugo, Josep Bayona y Lorenzo Morales (2014). «Microcontaminantes orgánicos en los ríos de México: El caso del río Nexapa». En M. Ramos y V. Aguilera (editores), Ciencias de la ingeniería y tecnología. Handbook T-IV 49-63). Valle de Santiago: Ecorfan.

ONU, Organización de las Naciones Unidas (2002). Observación General número 15. El derecho al agua (artículos 11 y 12 del Pacto Internacional de Derechos Eco- 
nómicos, Sociales y Culturales), documento E/C.12/2002/11, Consejo Económico y Social.

-. (2010). Resolución A/RES/64/292. El derecho humano al agua y el saneamiento, Asamblea General.

OPS, Organización Panamericana de la Salud (2012). Agua y saneamiento: En la búsqueda de nuevos paradigmas para las Américas, Washington: OPS.

Ramírez Cortina, Clementina (1992). Tratamiento de aguas residuales industriales. México: Universidad Autónoma Metropolitana, Azcapotzalco.

Robledo-Zacarías, Víctor Hugo, Martha A. Velázquez-Machuca, José L. Montañez-Soto, José L. Pimentel-Equihua, Alba Vallejo-Cardona, María D. López-Calvillo, y José Venegas-González (2017). «Hidroquímica y contaminantes emergentes en aguas residuales urbano industriales de Morelia, Michoacán, México». Revista Internacional de Contaminación Ambiental, 33 (2): 221-235. DOI: 10.20937/ RICA.2017.33.02.04.

Rocha-Gutiérrez, Beatriz A., María R. Peralta-Pérez y Francisco Zavala-Díaz (2015). «Revisión global de los contaminantes emergentes PBDE y el caso particular de México». Revista Internacional de Contaminación Ambiental, 31 (3): 311-320. SARH, Secretaría de Agricultura y Recursos Hidráulicos (1976). Uso del agua y manejo del agua residual en las industrias: petrolera, química, textil, celulosa y papel, minerales no metálicos, alimenticia, curtiduría, hierro y acero, acabado de metales, beneficio del café, vitivinícola y del azúcar. México: SARH.

Tadeo, José L., Consuelo Sánchez, Beatriz Albero, Ana I. García y Rosa A. Pérez. (2012). "Analysis of emerging organic contaminants in environmental solid samples». Central European Journal of Chemistry, 10 (3): 480-520. DOI: 10.2478/ s11532-011-0157-9.

USEPA, United States Environmental Protection Agency (2014). «Technical fact sheet polybrominated diphenyl ethers (PBDEs) and polybrominated biphenyls (PBBs)». Washington: Office of Solid Waste and Emergency Response.

USGS, United States Geological Survey (2014). «Emerging contaminants in the environment». Consultado el 26 de mayo de 2020. Disponible en https://pubs.usgs. gov/sir/2014/5096/pdf/sir2014-5096.pdf.

\section{Sobre los autores}

Daniel Jacobo-Marín es profesor de Derecho de Aguas. Abogado ambiental e integrante de la Clínica de Litigio Estratégico en Derechos Humanos de la Universidad Autónoma de San Luis de Potosí, México. Doctor en Derecho por la Facultad de Ciencias Sociales y Jurídicas de la Universidad de Jaén, España. Su correo electrónico es jacobo.marind@gmail.com. (D) https://orcid.org/0000-0001-8379-1220. 
Germán Santacruz de León es profesor investigador en el Programa Agua y Sociedad de El Colegio de San Luis. Doctor en Ciencias Ambientales por el Programa Multidisciplinario de Posgrado en Ciencias Ambientales de la Universidad Autónoma de San Luis Potosí, México. Su correo electrónico es german.santacruz@colsan. edu.mx. (D) https://orcid.org/00oo-0001-5231-6355. 
La Revista de Derecho Ambiental, del Centro de Derecho Ambiental de la Facultad de Derecho de la Universidad de Chile, es un espacio de exposición y análisis en el plano académico del derecho ambiental. Su contenido se presenta a través de doctrina, jurisprudencia y recensiones, y aborda diversas materias relacionadas con la gestión, institucionalidad y herramientas de protección ambiental y desarrollo sustentable. Se presentan artículos de diferentes autores y autoras en los que se analizan y abordan casos y temas jurídico-ambientales de creciente interés y actualidad.

\author{
DIRECTORA \\ Valentina Durán Medina \\ EDITORES \\ Jorge Ossandón Rosales \\ y Antonio Pulgar Martínez \\ SITIO WEB \\ revistaderechoambiental.uchile.cl \\ CORREO ELECTRÓNICO \\ revistada@derecho.uchile.cl \\ LICENCIA DE ESTE ARTÍ́CULO \\ Creative Commons Atribución Compartir Igual 4.o Internacional
}

La edición de textos, el diseño editorial

y la conversión a formatos electrónicos de este artículo

estuvieron a cargo de Tipográfica

(www.tipografica.io) 